\title{
Acute Care Utilization by Patients After Graduation of Their Resident Primary Care Physicians
}

\author{
Sonja R. Solomon, $M D^{1,2}$, Holly C. Gooding, MD, MS ${ }^{2,3}$, Harry Reyes Nieva, $B A^{1,2}$, \\ and Jeffrey A. Linder, MD, MPH ${ }^{1,2}$
}

'Division of General Internal Medicine \& Primary Care, Brigham and Women's Hospital, Boston, MA, USA; ${ }^{2}$ Harvard Medical School, Boston, MA, USA; ${ }^{3}$ Division of Adolescent and Young Adult Medicine, Boston Children's Hospital, Boston, MA, USA.

BACKGROUND: The disruption in provider continuity caused by medical resident graduation may result in adverse patient outcomes.

OBJECTIVE: Our aim was to investigate whether resident graduation was associated with increased acute care utilization by residents' primary care patients.

DESIGN AND PARTICIPANTS: This was a retrospective cohort study of patients cared for by junior and senior residents finishing the academic year in 2010, 2011 and 2012.

MAIN MEASURES: We compared rates of clinic visits, emergency department (ED) visits, and hospitalizations between transitioning patients whose residents were graduating and non-transitioning patients whose residents were not graduating.

KEY RESULTS: Our study population comprised 90 residents, 4018 unique patients, and 5988 resident-patient dyads that transitioned $(n=3136)$ or did not transition $(n=$ 2852). For transitioning patients, the clinic visit rate per 100 patients in the 4 months before and after graduation was 129 and 102, respectively; for non-transitioning patients, the clinic visit rate was 119 and 94, respectively (difference-in-differences, +2 per 100 patients; $p=0.12$ ). For transitioning patients, the ED visit rate per 100 patients before and after graduation was 29 and 26, respectively; for non-transitioning patients, the $\mathrm{ED}$ visit rate was 28 and 25, respectively (difference-in-differences, 0; $p=0.49$ ). For transitioning patients, the hospitalization rate per 100 patients before and after graduation was 14 and 13, respectively; for non-transitioning patients, the hospitalization rate was 15 and 12 , respectively (difference-in-differences, $-2 ; p=0.20$ ). In multivariable modeling there was no increased risk for transitioning patients for clinic visits (adjusted rate ratio [aRR], 1.03; $95 \%$ confidence interval [CI], 0.97 to 1.10), ED visits (aRR, 1.05; $95 \% \mathrm{CI}, 0.92$ to 1.20 ), or hospitalizations (aRR, 1.04; $95 \%$ CI, 0.83 to 1.31 ).

CONCLUSIONS: Acute care utilization by residents' patients did not increase or decrease after graduation. Acute care utilization was high before and after graduation. Interventions to decrease the need for acute care should be employed throughout the year.

Received December 9, 2014

Revised February 24, 2015

Accepted March 16, 2015

Published online April 21, 2015
KEY WORDS: continuity of care; care transitions; medical educationsystems based practice; medical education-graduate; ambulatory care; utilization.

J Gen Intern Med 30(11):1611-7

DOI: $10.1007 /$ s11606-015-3305-7

(C) Society of General Internal Medicine 2015

\section{BACKGROUND}

Continuity of care is a cornerstone of high-quality primary care. ${ }^{1}$ Increasing continuity with an individual provider is associated with increased rates of guideline-concordant preventative care, ${ }^{2}$ lower rates of hospitalization and emergency department (ED) utilization, ${ }^{3,4}$ lower cost of care for patients with chronic disease, ${ }^{5}$ and lower mortality among older adults. ${ }^{6}$ However, it is unknown whether patients are more vulnerable to adverse outcomes in the period immediately following the transition to a new primary care provider.

One substantial disruption to continuity occurs at the end of each academic year, when resident physicians graduate and transition the care of their primary care patients to new residents. Resident graduation affects approximately 1 million patients annually, and is particularly challenging due to the discrepancy of experience between outgoing and incoming physicians and the administrative complexity caused by the simultaneous departure of many clinicians. ${ }^{7}$ Many patients in teaching practices are subject to this particular transition of care repeatedly, and may be at risk for adverse outcomes associated with discontinuity of care.

Previous studies found lapses in care for the patients of graduating residents, including lack of follow-up of highpriority patients. ${ }^{8,9}$ Unknown is whether graduating residents' patients experience more complications or acute care utilization in the period following graduation compared to the period before graduation, and compared to non-graduating residents' patients. ${ }^{10}$ We conducted a retrospective cohort study to address whether acute care utilization, as a proxy for inadequate outpatient continuity of care and access, is increased among patients of graduating residents compared to non-graduating residents. 


\section{METHODS}

\section{Setting and Study Population}

The study practice is the largest of 19 clinics that serve as continuity practice sites for internal medicine residents at Brigham and Women's Hospital. We limited the study to this hospital-based, academic practice to examine a single, diverse, patient population. Other teaching practices have fewer residents and variable degrees of panel-sharing with faculty preceptors. Several other sites do not admit patients preferentially to Brigham and Women's Hospital, which would limit outcome ascertainment. At the study practice, residents serve as the primary care physician (PCP) for patients, with faculty precepting and supervision. Residents serve as the PCP for approximately $16 \%$ of patients in the practice. Residents' patients are uniformly insured through Medicare or Medicaid. Each year, 20 to 30 residents graduate and transition approximately 2000 patients to new resident PCPs. Most patients transition from graduating residents to first-year resident interns, but patient panels are not transitioned en masse from one resident to another, and for the majority of patients, there is no face-to-face meeting between the outgoing and incoming PCP. All transitioning patients receive written notification of the need to select a new PCP several months before graduation. Patients identified by their residents as high-risk are tracked by clinic staff to ensure that timely follow-up with a new PCP is scheduled. Graduating residents are instructed to sign out pending results to their preceptors.

Our study population comprised the patients of internal medicine junior and senior residents who finished their academic year in 2010, 2011 or 2012. We excluded residents with non-traditional training pathways (for example, a 2-year "short-track" into a medical subspecialty) and residents who remained as primary care faculty and retained their patients. Study residents included those in a traditional categorical track (20 weeks of dedicated ambulatory training over 3 years), a primary care track (40 weeks over 3 years), and a global health track (14 weeks over 3 years). All residents see patients in their continuity clinic approximately once weekly while on inpatient rotations, and 2-3 times weekly while on ambulatory rotations. The study was approved by the Partners HealthCare Human Research Committee.

\section{Defining the Primary Care Relationship}

A record of change in PCP over time for each patient was unavailable. Therefore, using billing and electronic health record (EHR) data, we developed an assignment algorithm to match each patient with a resident PCP. We defined a patient as having a given resident as their PCP if the patient had at least two visits with the resident in the 2 years before the annual date of graduation (June 20), and had seen that resident more than any other provider in the clinic in those 2 years. In the event of a tie in number of visits between clinicians, patients were assigned to the clinician who saw them closest to the graduation date.

We used a two-year primary care relationship interval to maintain an exposure period that was consistent for patients of senior (graduating) and junior (non-graduating) residents. We excluded patients of residents completing their first year of training to avoid the confounding effects of provider inexperience and a shorter-term patient-physician relationship.

We validated the PCP assignments through chart review of $10 \%$ of randomly sampled transitioning patient charts $(n=$ 314). We looked for language reflecting that the visit was conducted by the PCP, either in the title or text of the note. If the note was labeled "Urgent Care Visit," the documentation reflected that the patient's PCP was another provider, or it was unclear that the visit was conducted by the assigned PCP, the visit-based assignment was considered incorrect. Based on chart review, our visit-based definition had a positive predictive value of $99 \%$. The remaining $1 \%$ were patients seen most by a resident in the preceding two years for urgent care visits.

\section{Defining Transitioning and Non-Transitioning Patient-Resident Dyads}

We identified patients with visits to the study practice between 20 June 2008 and 20 June 2012. From this cohort, we assigned patients to one or more residents using the definition above. For each transition year (2010, 2011 and 2012), we defined "transitioning dyads," patients associated with senior residents who were graduating; and "nontransitioning dyads," patients associated with junior residents completing their second year of training and entering their final year. Using this approach, we created six study groups (Groups A-F; Fig. 1). Residents generally appeared in the study twice, when they concluded their junior year and when they concluded their senior year. A patient could appear in the study multiple times, linked to the same or different residents for different years.

\section{Acute Care Utilization Outcomes}

Using billing data, we calculated the number of visits to the study practice, emergency department (ED) visits, hospitalizations, and hospitalizations for ambulatory care sensitive conditions (ACSCs) in the 4 months before and 4 months after 20 June for each patient, for each year under study. A clinic visit was defined as any medical visit to the study clinic with any provider, regardless of whether that provider was the PCP or an urgent care provider. An ED visit was defined as any visit to the ED, regardless of indication, and regardless of whether it resulted in admission. A hospitalization was defined as an inpatient admission of any duration for any indication. An ACSC hospitalization was defined according to criteria established by the Agency for Healthcare Research and Quality. ${ }^{11}$ ED visits and hospitalizations were considered to be adverse outcomes, while visits to the study practice were considered neutral outcomes reflecting patients' ability to 


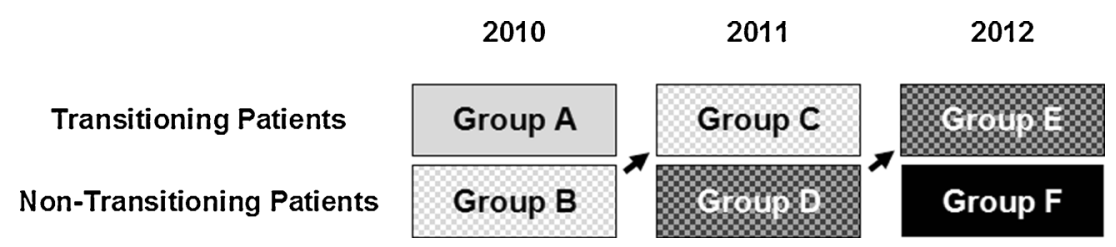

Figure 1. Description of transitioning and non-transitioning patient groups. Groups A, C and E: Patients of senior residents graduating in 2010, 2011 and 2012, respectively. Groups B, D and F: Patients of junior residents entering their final year of training in 2010, 2011 and 2012, respectively. Arrows and shading denote that most residents and many patients in Groups B and D will become residents, and patients in Groups $\mathrm{C}$ and $\mathrm{E}$, respectively.

access the clinic for care at different time points. We chose an interval of 4 months before and after the transition date to capture acute care episodes that were proximate to the transition date and represent an accurate baseline of ongoing acute care utilization prior to the transition date, and to avoid the winter months where we would expect to see an increase in acute care utilization unrelated to a change in PCP. This follow-up interval is similar to that used in a prior study of this transition.

\section{Covariates and Data Analysis}

We extracted patient sociodemographic information from the EHR. Our system conflated race and ethnicity, which we grouped into the categories of White, Black, Latino, Asian/ Pacific Islander, and Other. We derived a comorbidity score using a combination of coded EHR problem list entries and International Classification of Diseases-9, Clinical Modification (ICD-9) billing codes. We selected comorbidities based on their clinical significance and inclusion in the Charlson Comorbidity Index (Table 1). ${ }^{12}$ For each patient, we also calculated the usual provider continuity (UPC) index, the fraction of total clinic visits with the patient's PCP during the 2-year review period that preceded the resident's graduation date.

\section{Statistical Analysis}

To examine differences between transitioning and nontransitioning resident-patient dyads, we used Fisher's Exact Test and the Chi-squared test for categorical variables and Student's $t$ test for continuous variables. We calculated incident rates for each of the four care utilization outcomes. We assessed the difference in rates within transitioning and non-transitioning patient groups using negative binomial regression.

To assess the independent association between transitioning and clinic visits, ED visits, and hospitalizations, we used multivariable negative binomial regression. The rate of ACSC hospitalizations was too low to reliably examine using multivariable modeling. We adjusted for potential confounders related to acute care utilization including patient factors (age, sex, race/ethnicity, and pre-transition incident rates), resident factors (categorical, primary care or global health training track), and clustering by patients. The latter adjusted for the effect of patients appearing in multiple groups in different years. Neither additional adjustment for clustering by resident nor use of zero-inflated negative binomial regression changed the results substantively.

\section{RESULTS}

\section{Patients and Residents}

Ninety-nine residents with continuity clinic in the study practice graduated in the years 2010-2013 (Fig. 2). We excluded nine residents: seven had non-traditional training pathways and two became primary care faculty who retained their primary care patients. Of the 90 remaining residents, 65 were in the categorical track, 15 were in primary care, nine were in global health, and one resident was in both the primary care and global health tracks.

Of approximately 122,000 patients seen in the study practice between 2008 and 2012, 4018 unique patients met our criteria for belonging to one or more residents' primary care panels, resulting in 5988 resident-patient dyads. Of these, 3136 dyads transitioned (i.e., the senior resident graduated; 961 in 2010, 1092 in 2011, and 1083 in 2012) and 2852 dyads did not transition (i.e., the junior resident advanced to their final year of training; 884 in 2010, 951 in 2011, and 1017 in 2012; Fig. 2). Within dyads, the average number of patients associated with each resident was 67 . Of the 5988 dyads, 354 $(5.9 \%)$ had a tie in PCP by our algorithm and the patients were assigned to the resident with the visit closest to graduation.

Patients had a mean age of 52 years; were $67 \%$ women; $44 \%$ Black, $26 \%$ Latino, and $23 \%$ White; and $76 \%$ spoke English and $14 \%$ spoke Spanish (Table 1). The mean UPC index was 0.7 (range, 0.1 to 1.0 ) prior to the date of transition, and did not differ between transitioning and non-transitioning patients. Transitioning patients were slightly older, made slightly more visits with their resident in the 2 years prior to the transition, had higher comorbidity scores, and were more likely to have categorical resident PCPs.

\section{Acute Care Utilization Outcomes}

In the 4-month pre-transition and post-transition periods, the proportion of patients who had any clinic visit was 69 and $53 \%$, respectively; the proportion of patients who had ED visits was 18 and $17 \%$, respectively. The proportion of patients who had hospitalizations was $8 \%$ in both the pretransition and post-transition periods and the rate of ACSC hospitalizations was $2 \%$ in both the pre-transition and posttransition periods.

Rates of clinic visits were significantly higher in the pretransition period than in the post-transition period. There was a 
Table 1. Patient Demographics

\begin{tabular}{|c|c|c|c|c|}
\hline Characteristic & Overall $(n=5988)$ & Non-transitioning $(n=2852)$ & Transitioning $(n=3136)$ & $p$ value \\
\hline & \multicolumn{4}{|c|}{ Mean (Standard deviation) } \\
\hline Age, years & $52(17)$ & $51(17)$ & $53(17)$ & $<0.001$ \\
\hline Clinic visits with resident & $4.2(2.2)$ & $4.0(2.1)$ & $4.3(2.3)$ & $<0.001$ \\
\hline UPC index & $0.7(0.2)$ & $0.7(0.2)$ & $0.7(0.2)$ & 0.85 \\
\hline \multirow[t]{2}{*}{ Comorbidity score } & $0.8(1.7)$ & $0.7(1.5)$ & $0.9(1.9)$ & 0.01 \\
\hline & \multirow{2}{*}{\multicolumn{3}{|c|}{$\mathrm{N}(\%)$}} & \\
\hline Gender & & & & 0.81 \\
\hline Women & $4015(67)$ & $1908(67)$ & $2107(67)$ & \\
\hline \multirow{2}{*}{\multicolumn{4}{|c|}{ Race/Ethnicity }} & \\
\hline & & & & 0.20 \\
\hline Black & $2625(44)$ & $1223(43)$ & $1402(45)$ & \\
\hline Latino & $1565(26)$ & $733(26)$ & $832(27)$ & \\
\hline White & $1402(23)$ & $706(25)$ & $696(22)$ & \\
\hline Asian/Pacific Islander & $174(3)$ & $86(3)$ & $88(3)$ & \\
\hline Other & $222(4)$ & $104(4)$ & $118(4)$ & \\
\hline \multicolumn{4}{|l|}{ Primary language } & 0.26 \\
\hline English & $4641(76)$ & $2237(78)$ & $2404(77)$ & \\
\hline Spanish & $854(14)$ & 389 (14) & $465(15)$ & \\
\hline Other & $493(8)$ & $226(8)$ & $267(9)$ & \\
\hline \multicolumn{4}{|l|}{ Resident type } & $<0.001$ \\
\hline Categorical & $4055(68)$ & $1834(64)$ & $2221(71)$ & \\
\hline Primary care & $1373(23)$ & $725(25)$ & $648(21)$ & \\
\hline Global health & $465(8)$ & $242(8)$ & $223(7)$ & \\
\hline Primary care and global health & $95(2)$ & $51(2)$ & $44(1)$ & \\
\hline \multicolumn{5}{|l|}{ Comorbidities } \\
\hline Cancer (Metastatic) & $44(1)$ & $18(1)$ & $26(1)$ & 0.45 \\
\hline Cancer (Non-metastatic) & $342(6)$ & $151(5)$ & $191(6)$ & 0.20 \\
\hline Cerebrovascular disease & $197(3)$ & $90(3)$ & $107(3)$ & 0.61 \\
\hline Chronic kidney disease (Moderate/Severe) & $95(2)$ & $46(2)$ & $49(2)$ & 0.92 \\
\hline Chronic obstructive pulmonary disease & $723(12)$ & $322(11)$ & $401(13)$ & 0.08 \\
\hline Congestive heart failure & $328(5)$ & $142(5)$ & $186(6)$ & 0.11 \\
\hline Connective tissue disease & $222(4)$ & $95(3)$ & $127(4)$ & 0.15 \\
\hline Dementia & $61(1)$ & $30(1)$ & $31(1)$ & 0.90 \\
\hline Diabetes & $1236(21)$ & $548(19)$ & $688(22)$ & 0.009 \\
\hline Hemiplegia & $28(11)$ & $11(<1)$ & $17(1)$ & 0.45 \\
\hline HIV/AIDS & $57(1)$ & $27(1)$ & $30(1)$ & 0.99 \\
\hline Myocardial infarction & $180(3)$ & $71(2)$ & $109(3)$ & 0.03 \\
\hline Liver disease (Mild) & $79(1)$ & $34(1)$ & $44(1)$ & 0.50 \\
\hline Liver disease (Moderate/Severe) & $31(1)$ & $12(<1)$ & $19(1)$ & 0.37 \\
\hline Peptic ulcer disease & $58(1)$ & $21(1)$ & $37(1)$ & 0.09 \\
\hline Peripheral vascular disease & $99(2)$ & $47(2)$ & $52(2)$ & 0.99 \\
\hline
\end{tabular}

The sample represents 90 residents, 4018 patients, and 5988 resident-patient dyads

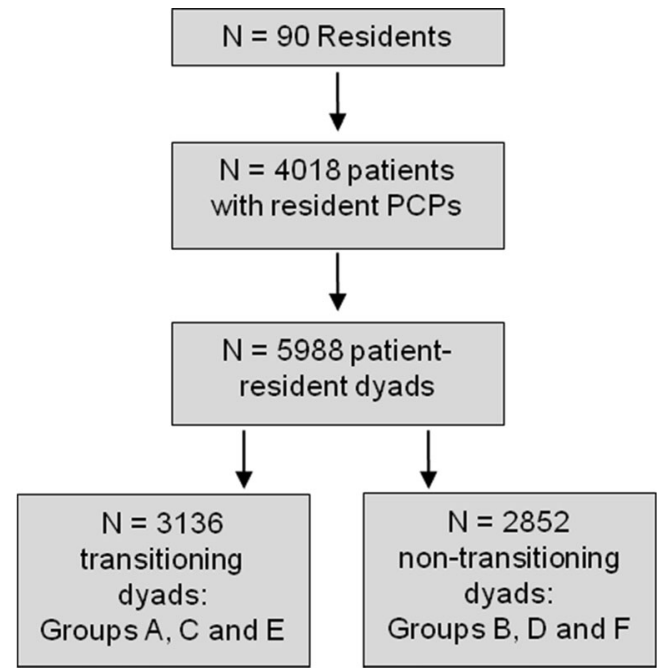

Figure 2. Creation of transitioning and non-transitioning dyads. On chart review, the PCP assignment algorithm had a $99 \%$ positive predictive value for a primary care relationship. Over the 3 years of study, 3461 patients had one resident PCP, 552 had two resident PCPs, and five patients had three resident PCPs. significant decrease in the rate of hospitalizations for nontransitioning patients. Overall, transitioning patients had no increase or decrease in the rate of clinic visits, ED visits, hospitalizations, or ACSC hospitalizations compared to nontransitioning patients (Table 2).

In multivariable modeling, transitioning patients had no increased risk of clinic visits (adjusted odds ratio [aOR], 1.03; $95 \%$ confidence interval [CI], 0.97 to 1.09 ), ED visits (aOR, 1.03; $95 \% \mathrm{CI}, 0.90$ to 1.17 ), or hospitalizations (aOR, 1.10; $95 \% \mathrm{CI}, 0.87$ to 1.40; Table 3). Independent predictors of clinic visits, ED visits, and hospitalizations were higher pre-transition visit rates and higher comorbidity scores.

\section{DISCUSSION}

The graduation of resident physicians represents an annual, large-scale disruption in primary care continuity. This disruption could leave patients vulnerable to adverse outcomes. 
Table 2. Utilization Rates for Transitioning vs. Non-Transitioning Patients

\begin{tabular}{|c|c|c|c|c|c|c|}
\hline \multirow[b]{2}{*}{ Encounter type } & & \multicolumn{2}{|c|}{$\begin{array}{l}\text { Rate per } 100 \text { patients } \\
\text { per } 4 \text {-month period }\end{array}$} & \multirow[b]{2}{*}{$\begin{array}{l}\text { p value pre-post } \\
\text { difference }\end{array}$} & \multirow[b]{2}{*}{$\begin{array}{l}\Delta \text {-in- } \Delta \text { 's between } \\
\text { transitioning and } \\
\text { non-transitioning }\end{array}$} & \multirow[b]{2}{*}{$\underset{\Delta \text {-in- } \Delta^{*}}{p \text { value }}$} \\
\hline & & Pre & Post & & & \\
\hline \multirow{2}{*}{ Clinic visits } & Transitioning & 129 & 102 & $<0.001$ & 2 & 0.12 \\
\hline & Non-transitioning & 119 & 94 & $<0.001$ & & \\
\hline \multirow[t]{2}{*}{ ED Visits } & Transitioning & 29 & 26 & 0.14 & 0 & 0.49 \\
\hline & Non-transitioning & 28 & 25 & 0.05 & & \\
\hline \multirow[t]{2}{*}{ Hospitalizations } & Transitioning & 14 & 13 & 0.28 & -2 & 0.23 \\
\hline & Non-transitioning & 15 & 12 & 0.04 & & \\
\hline \multirow[t]{2}{*}{ ACSC hospitalizations } & Transitioning & 4 & 3 & 0.05 & 1 & 0.19 \\
\hline & Non-transitioning & 3 & 3 & 0.90 & & \\
\hline
\end{tabular}

${ }^{*} p$ value is difference between pre and post rates assessed by negative binomial regression

$\Delta$-in- $\Delta$ is difference-in-differences

${ }^{*} p$ value of transitioning based on negative binomial regression modeling, adjusted for the baseline rate and clustering by patient

Other studies have described this vulnerability by retrospectively examining cohorts of patients whose residents recently graduated, assessing rates of loss-to-follow-up, inadequate follow-up of abnormal test results, missed preventive screening opportunities, and acute care utilization. ${ }^{8,9}$

Our study represents the first attempt to utilize a control group of non-transitioning patients to assess transitionrelated outcomes. We hypothesized that resident graduation would be associated with an increase in ED visits and hospitalizations. To the contrary, we found that the rate of clinic visits, ED visits, hospitalizations, and ACSC hospitalizations did not change after resident graduation for transitioning patients. The effect of provider transitioning status had no bearing on pre-post differences in utilization rates.

However, we observed a high rate of acute care utilization in all time periods examined. We observed 25 to $29 \mathrm{ED}$ visits per 100 patients per 4-month interval and 12 to 15 hospitalizations per 100 patients per 4-month interval (Table 2). These rates extrapolate to annual rates of 75-97 ED visits per 100 patients and 36-45 hospitalizations per 100 patients. These are much higher than national averages, which for the year 2010 were 43 ED visits per 100 people per year ${ }^{13}$ and 11 hospital discharges per 100 people per year. ${ }^{14}$

In this study, we did not determine what factors account for the observed high rates of acute care utilization, but hypothesize that medical complexity, low socioeconomic status and lack of awareness about clinic resources for managing acute issues may all play a role. Of note, adjusting for clustering by resident did not alter our results, arguing against the possibility that individual residents providing either better or worse care would confound the outcomes.

With respect to clinic visits, we observed a lower rate of visits to the clinic in the 4 months following graduation than in the 4 months prior. This is likely multifactorial: graduating senior residents may make an effort to schedule final visits with patients before their departure; visits to clinic likely decline during the summer months; and access to residents' clinics is more limited post-transition due to fewer appointments per session for new trainees compared to experienced trainees. The fact that the pre-post differences were similar between transitioning and nontransitioning patients argues that the transition itself was not the cause of this difference.

Table 3. Multivariable Models of Post-Transition Acute Care Utilization

\begin{tabular}{|c|c|c|c|}
\hline \multirow[b]{2}{*}{ Characteristic } & \multicolumn{3}{|c|}{ Odds ratio (95\% confidence interval) } \\
\hline & Clinic visits & $\begin{array}{l}\text { Emergency department } \\
\text { visits }\end{array}$ & $\overline{\text { Hospitalizations }}$ \\
\hline Transitioning & $1.03(0.97-1.09)$ & $1.03(0.90-1.17)$ & $1.10(0.87-1.40)$ \\
\hline Pre-transition visits, per visit in the 4 months prior to transition & $1.33(1.30-1.36)$ & $1.83(1.75-1.91)$ & $1.98(1.87-2.11)$ \\
\hline Comorbidity score, per point & $1.07(1.05-1.08)$ & $1.16(1.13-1.19)$ & $1.33(1.29-1.37)$ \\
\hline Patient age, per decade & $1.09(1.06-1.11)$ & $0.95(0.91-0.99)$ & $1.04(0.97-1.12)$ \\
\hline Women, versus men & $1.13(1.05-1.21)$ & $0.98(0.84-1.13)$ & $1.02(0.78-1.32)$ \\
\hline \multicolumn{4}{|l|}{ Race/ethnicity, compared to Whites } \\
\hline African American/Black & $1.17(1.07-1.29)$ & $1.13(0.92-1.38)$ & $1.06(0.78-1.45)$ \\
\hline Asian/Pacific Islander & $1.06(0.88-1.28)$ & $0.60(0.38-0.94)$ & $0.50(0.24-1.03)$ \\
\hline Latino/Hispanic & $1.24(1.13-1.37)$ & $1.23(0.99-1.52)$ & $0.75(0.52-1.08)$ \\
\hline Other/Unknown & $1.09(0.91-1.30)$ & $0.68(0.41-1.10)$ & $0.92(0.40-2.11)$ \\
\hline \multicolumn{4}{|l|}{ Resident type, compared to categorical } \\
\hline Primary care & $1.03(0.96-1.11)$ & $1.06(0.90-1.25)$ & $1.06(0.80-1.41)$ \\
\hline Global health & $0.99(0.88-1.12)$ & $1.05(0.83-1.34)$ & $1.01(0.67-1.54)$ \\
\hline Primary care and global health & $1.03(0.81-1.32)$ & $1.04(0.65-1.69)$ & $1.76(0.83-3.74)$ \\
\hline
\end{tabular}

There was an insufficient rate of ACSC hospitalizations to reliably model. Usual provider continuity index and resident visits, which were colinear with pre-transition visit rates, were not included in the models. Patient language, which was colinear with race, was not included in the models. 
There were several limitations of our study. First, our analysis was restricted to a single clinic site and may not be generalizable to other settings. Second, we were unable to capture episodes of acute care occurring outside the institutions included in our EHR. However, we would not expect the rate of ED visits or hospitalizations at outside institutions to be differentially affected by the transition. Third, we did not examine other outcomes that may reflect inadequate transitions of care including chronic disease-specific metrics, ontime medication refills, follow-up of abnormal results, or patient satisfaction.

Finally, a limitation in any study of care continuity is the way in which the linkage between patient and primary care provider is defined. In our study, we chose a definition based on clinical encounters, rather than the PCP listed in the EHR, as the latter was not available retrospectively. Patients were assigned to a resident PCP only if they had seen that resident for two or more visits in the previous 2 years and had seen no other clinic provider more than that resident. We therefore excluded patients who were less closely linked to a single physician. Excluded patients could be at either increased risk (e.g., due to less continuity) or decreased risk (e.g., due to lack of medical complexity) of acute care utilization. However, our method of linking patients to a resident PCP was balanced between the patients of graduating and non-graduating residents, and introduced a conservative bias for our estimates: we would expect patients with more individual continuity to be more influenced by the graduation of their PCP. Despite this bias, we saw no association between resident graduation and increased acute care utilization.

On the other hand, it could be argued that the length and closeness of the primary care relationships in this study are in fact insufficient to cause significant alterations in care utilization when they terminate. More research is needed to determine whether the loss of a more long-standing primary care relationship is associated with acute care utilization or adverse clinical outcomes.

Our study demonstrates that residents care for a population at high risk of acute care utilization, and interventions to reduce inappropriate acute care utilization should be employed throughout the academic year. Such interventions might include enhancing patient education about how to access their care team for urgent needs; developing care management strategies for high-risk patients; and identifying patients with frequent ED visits or hospitalization for targeted intervention. These initiatives have all been undertaken to various degrees at our institution and further research will be necessary to measure their effects. We acknowledge that our study does not answer the question of whether achieving a certain threshold of ambulatory continuity will decrease acute care utilization by residents' patients, and if so, what that threshold might be. It would be worthwhile to examine whether demographically similar patients cared for by attendings in our practice exhibit different rates of acute care utilization, and if so, whether the duration of the primary care relationship is predictive.
We continue to support the development and implementation of standardized procedures for transitioning the care of ambulatory patients at the end of each academic year, as such procedures make good sense clinically and operationally. A standardized process may benefit other members of the care team, including clinic staff and residents themselves, who selfreport anxiety surrounding the year-end transition ${ }^{9}$; such a process has been shown to improve patients' rate of followup with their new PCP. ${ }^{15}$

In conclusion, we found that resident graduation from a large teaching practice did not impact their patients' acute care utilization. However, high rates of acute care utilization before and after the transition highlight the need to better understand this problem and develop targeted interventions to address it throughout the academic year.

Acknowledgements:

Contributors: The authors would like to acknowledge Stuart Lipsitz, $\mathrm{ScD}$ and Robert Glynn, PhD for biostatistical consultation and Edward Carreras, MD for assistance with chart review.

Funders: This work was funded by a Support for Excellence in Educational Development (SEED) Grant from the Brigham and Women's Hospital Department of Medicine.

Prior Presentations: Presented in part at the Society of General Internal Medicine New England Regional Meeting, 7 March 2014, Boston, MA and the Society of General Internal Medicine Annual Meeting, 24 April 2014, San Diego, CA.

Conflicts of Interest: The authors have no potential conflicts of interest.

Corresponding Author: Sonja R. Solomon, MD; Division of General Internal Medicine \& Primary Care, Brigham and Women's Hospital, 1620 Tremont Street, BC-3, Boston, MA 02120, USA (e-mail: ssolomon5@partners.org).

\section{REFERENCES}

1. Bodenheimer T, Chorob A, Willard-Grace R, Grumbach K. The 10 building blocks of high-performing primary care. Ann Fam Med. 2014;12(2): 166-71.

2. Atlas SJ, Grant RW, Ferris TG, Chang Y, Barry MJ. Patient-physician connectedness and quality of primary care. Ann Intern Med. 2009; 150(5):325-35.

3. Nyweide DJ, Anthony DL, Bynum JP, Strawderman RL, Weeks WB, Casalino LP, et al. Continuity of care and the risk of preventable hospitalization in older adults. JAMA Intern Med. 2013;173(20):1879-85.

4. Chaiyachati $\mathbf{K H}$, Gordon $\mathbf{K}$, Long $\mathbf{T}$, et al. Continuity in a VA patientcentered medical home reduces emergency department visits. PLoS ONE. 2014;9(5):e96356.

5. Hussey PS, Schneider EC, Rudin RS, Fox DS, Lai J, Pollack CE. Continuity and the costs of care for chronic disease. JAMA Intern Med. 2014; 174(5):742-8.

6. Wilonsky FD, Bentler SE, Liu L, et al. Continuity of care with a primary care physician and mortality in older adults. J Gerntol A Biol Sci Med Sci. 2010;65(4):421-8.

7. Young JQ, Wachter RM. Academic year-end transfers of outpatients from outgoing to incoming residents: an unaddressed patient safety issue. JAMA. 2009;302(12):1327-9.

8. Caines LC, Brockmeyer DM, Tess AV, Kim H, Kriegel G, Bates CK. The revolving door of resident continuity practice: identifying gaps in transitions of care. J Gen Intern Med. 2011;6(9):995-8. 
9. Pincavage AT, Ratner S, Prochaska ML, et al. Outcomes for residentidentified high-risk patients and resident perspectives of year-end continuity clinic handoffs. J Gen Intern Med. 2012;27(11):1438-44.

10. Bump GM. Residents' final transition: the graduation clinic hand-off. J Gen Intern Med. 2015;30(2):145-6.

11. Agency for Healthcare Research and Quality. Quality Indicators Software Instruction, SAS Version 4.4. Available at: http://www.qualityindicators.ahrq. gov/Downloads/Software/SAS/V44/Software\%20Instructions\%20(SAS) \%20V\%204.4.pdf. Accessed March 5, 2015.

12. Charlson ME, Pompei P, Ales KL, MacKenzie CR. A new method of classifying prognostic comorbidity in longitudinal studies: development and validation. J Chronic Dis. 1987;40:373-83.
13. National Hospital Ambulatory Medical Care Survey: 2010 Emergency Department Summary Tables. Available at: http://www.cdc.gov/nchs/ data/ahcd/nhamcs_emergency/2010_ed_web_tables.pdf. Accessed March $5,2015$.

14. CDC/NCHS National Hospital Discharge Survey, 2010. Available at: http://www.cdc.gov/nchs/data/nhds/1general/2010gen1_agesexalos. pdf. Accessed March 5, 2015.

15. Pincavage AT, Lee WW, Venable LR. "Ms. B changes doctors": using a comic and patient transition packet to engineer patient-oriented clinic handoffs (EPOCH). J Gen Intern Med. 2015;30(2):257-60. 